\title{
Hiperhidrosis del cuero cabelludo: particularidades clínicas y de respuesta al tratamiento con oxibutinina oral
}

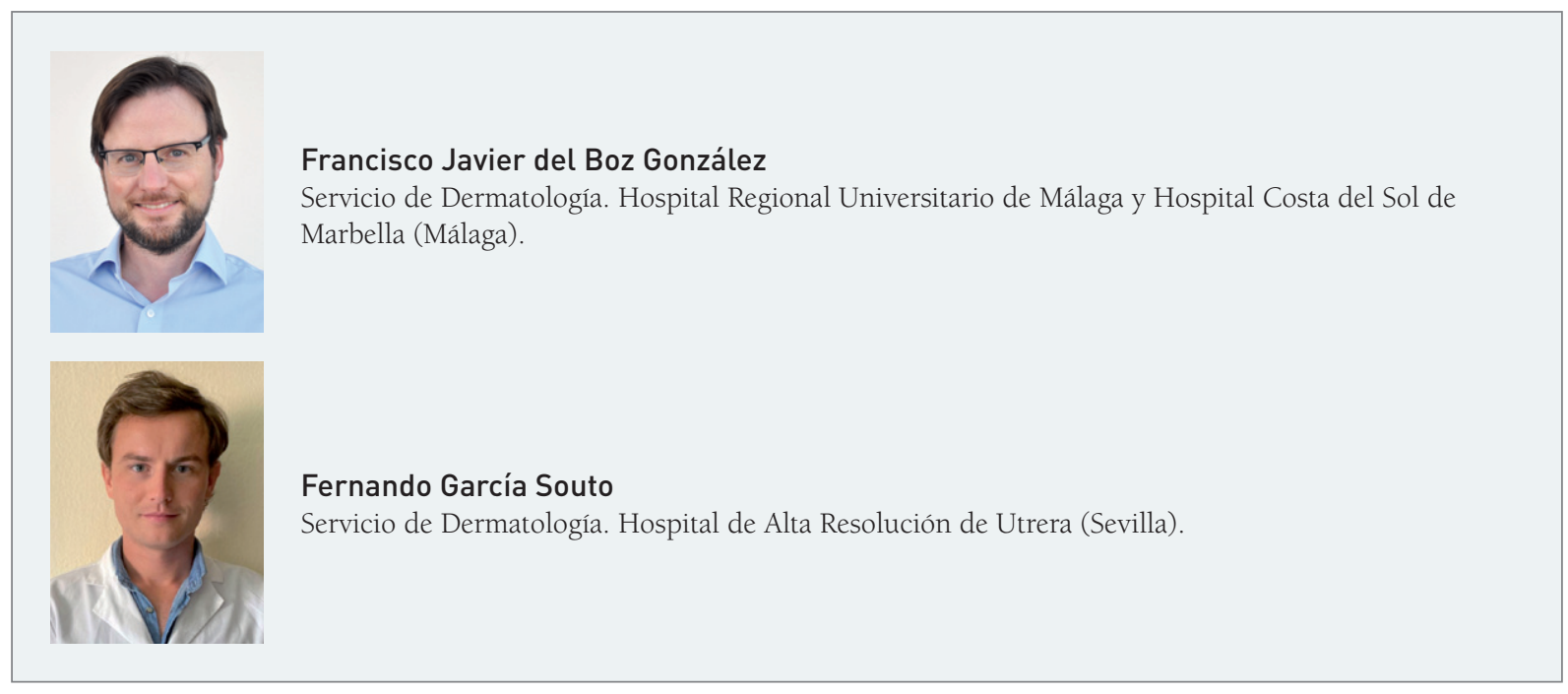

\section{INTRODUCCIÓN}

La hiperhidrosis del cuero cabelludo (HHCC) es un problema relativamente común y con gran impacto negativo en la calidad de vida de los pacientes ${ }^{1}$. Sin embargo, existen pocos estudios científicos centrados en esta ubicación específica y, de forma general, se limitan a hablar de hiperhidrosis craneofacial sin distinguir entre cara y/o cuero cabelludo ${ }^{1-6}$. Además, para esta localización especial, existen pocas alternativas terapéuticas disponibles, siendo los anticolinérgicos orales habitualmente considerados el tratamiento de elección y, concretamente, la oxibutinina oral $(\mathrm{OxO})$ el más usado ${ }^{7}$, si bien, no existen estudios centrados en valorar su uso específicamente en la HHCC.

El objetivo de nuestro estudio es describir las características clínicas de los pacientes con HHCC y compararlos con aquellos pacientes con hiperhidrosis sin afectación del cuero cabelludo (HHSCC).
Como objetivos secundarios, determinamos la efectividad y los efectos adversos de la $\mathrm{OxO}$, y evaluamos la adherencia de los pacientes a esta.

\section{MATERIAL Y MÉTODOS}

Se realizó un estudio observacional prospectivo entre los años 2007 y 2019. Se incluyó a todos los pacientes diagnosticados de HHCC y que fueron tratados con $\mathrm{OxO}$ en la Unidad de Hiperhidrosis del Hospital Costa del Sol (Málaga). Al tratarse de un uso fuera de la ficha técnica, los pacientes fueron informados previamente de sus posibles ventajas y efectos adversos y firmaron un consentimiento informado. La escala de gravedad de hiperhidrosis específica y validada Hyperhidrosis Disease Severity Scale $(\mathrm{HDSS})^{8}$ se utilizó para medir la intensidad de la sudoración y evaluar la respuesta al tratamiento. En esta, los valores de 3 y 4 corresponden a hiper- 
hidrosis grave; de 2, a moderada; y de 1 , a leve. La respuesta se definió como «excelente» cuando la puntuación en la HDSS postratamiento fue de 1 o si se redujo en, al menos, 2 puntos. Se calificó como «parcial» si se redujo en un punto, y se consideró «fallo terapéutico» si no hubo mejoría en la HDSS o si se produjo intolerancia a la $\mathrm{OxO}$. Se usaron los criterios diagnósticos propuestos por Walling para diferenciar entre hiperhidrosis primaria y secundaria ${ }^{9}$. El análisis descriptivo incluyó medidas de tendencia central para las variables cuantitativas y de distribución de frecuencias para las cualitativas. Para el contraste de hipótesis, se emplearon la prueba estadística de la chi cuadrado para las variables cualitativas y la prueba de la $U$ de Mann-Whitney para las cuantitativas. Además, se realizó un análisis de supervivencia para evaluar la adherencia al tratamiento. El nivel de significación estadística ( $p$ ) asumido fue $p<0,05$. Todos los análisis estadísticos se realizaron utilizando el programa SPSS v. 25 para macOS.

\section{RESULTADOS}

En las tablas 1 y 2 , se describen las características de los 292 pacientes tratados con $\mathrm{OxO}$, diferenciando los 41 pacientes (el 14\% del total) que presentaban HHCC del resto de pacientes (HHSCC). En la tabla 1, se representan las características epidemiológicas y clínicas basales de estos pacientes. En el grupo de HHCC, la media de edad fue 54,2 $( \pm 16,2)$ años, eran mujeres $27(65,9 \%)$, e igualmente 27 (65,9\%) habían comenzado a sufrir hiperhidrosis en la edad adulta. En 22 pacientes $(53,7 \%)$, la hiperhidrosis fue considerada secundaria, no presentaban antecedentes familiares de hiperhidrosis 25 pacientes (61\%), y se asoció a hiperhidrosis generalizada, en el tronco y en la cara, respectivamente, en 21 (51,2\%), 15 (36,6\%) y 37 $(90,2 \%)$ de los pacientes. Presentaron 34 pacientes $(82,9 \%)$ una puntuación en la HDSS basal de 4 .

Comparando ambos grupos, se apreciaron diferencias estadísticamente significativas en la media de edad de inicio de la hiperhidrosis, en el tipo de hiperhidrosis, en las áreas corporales afectadas de hiperhidrosis asociadas, y en el uso previo de otros tratamientos (antes del uso de OxO). En cambio, no se observaron diferencias estadísticamente significativas respecto al género de los pacientes, los antecedentes familiares de hiperhidrosis y la puntuación basal en la HDSS.

En la tabla 2, se representan la dosis media diaria de $\mathrm{OxO}$, la respuesta y los efectos secundarios de este tratamiento a los 3 y a los 12 meses de comenzarlo, y el motivo de suspensión. No se apreciaron diferencias estadísticamente significativas entre ambos grupos. Entre los pacientes con HHCC, la dosis media diaria de $\mathrm{OxO}$ fue de $7,7 \mathrm{mg}$ a los 3 meses de tratamiento y de 9,3 mg a los 12 meses, y la puntuación en la HDSS pre-OxO y tras 3 y 12 meses de tratamiento fue de $3,8,2,5$ y 1,4 , respectivamente. Se consideró la respuesta como excelente en 21 pacientes $(51,2 \%)$ a los 3 meses, y en $14(66,7 \%)$ a los 12 meses. Se registraron efectos adversos asociados en 29 pacientes $(70,7 \%)$ a los 3 meses, y en $17(81 \%)$ a los 12 meses y, concretamente, xerosis oral grave en 10 pacientes $(24,4 \%)$ a los 3 meses y en 6 pacientes (28,6\%) a los 12 meses. La razón de suspensión de la $\mathrm{OxO}$ fueron los efectos adversos en 9 casos $(21,9 \%)$, la ineficacia en 5 pacientes $(12,2 \%)$, ineficacia + efectos adversos en $7(17,1 \%)$ y decisión propia del paciente en otros 5 casos (12,2\%).

Respecto a la figura 1 (análisis de supervivencia de ambos grupos), se encontraron diferencias significativas entre ambas curvas de supervivencia (prueba de $\log$-rank $p=0,001$ ). En el grupo de pacientes con HHCC, la mediana de supervivencia fue de 35,95 meses frente a 61,35 meses en el grupo sin HHCC.

\section{DISCUSIÓN}

Los pacientes con HHCC - respecto a aquellos con HHSCC - presentaron, con diferencias estadísticamente significativas, una hiperhidrosis que debutó con más frecuencia a edades más tardías (en la edad adulta), comenzaron el tratamiento con $\mathrm{OxO}$ a una 
ORIGINAL

Hiperhidrosis del cuero cabelludo: particularidades clínicas y de respuesta al tratamiento con oxibutinina oral Del Boz González FJ et al.

\section{TABLA 1. Características basales clínicas y epidemiológicas de los pacientes}

\begin{tabular}{l|c|c|c|c} 
& $\begin{array}{c}\text { Total de pacientes } \\
\text { con HH tratados con } \\
\text { OxO } \\
\mathbf{n}=292\end{array}$ & $\begin{array}{c}\text { Pacientes con HHCC } \\
\mathbf{n}=41\end{array}$ & $\begin{array}{c}\text { Pacientes con } \\
\text { HHSCC } \\
\mathbf{n}=251\end{array}$ & $p$ \\
\hline $\begin{array}{l}\text { Media de edad } \pm \text { DE } \\
\text { (años) }\end{array}$ & $34,2 \pm 16,8$ & $54,2 \pm 16,2$ & $30,9 \pm 14,5$ & $<0,001$ \\
\hline & $\mathrm{n} \%$ & $\mathrm{n} \%$ & $\mathrm{n} \%$ & \\
\hline
\end{tabular}

\section{Sexo}

\begin{tabular}{l|c|c|c|c}
\hline Varón & $88(30,1)$ & $14(34,1)$ & $74(29,5)$ & 0,546 \\
\hline Mujer & $204(69,9)$ & $27(65,9)$ & $177(70,5)$ & \\
\hline
\end{tabular}

Edad de inicio de la $\mathrm{HH}$

\begin{tabular}{l|c|c|c|c}
\hline Infancia & $199(68,2)$ & $9(22)$ & $190(75,7)$ & $<0,001$ \\
\hline Adolescencia & $34(11,6)$ & $5(12,2)$ & $29(11,6)$ & \\
\hline Edad adulta & $59(20,2)$ & $27(65,9)$ & $32(12,7)$ & \\
\hline
\end{tabular}

\section{Tipo de $\mathrm{HH}$}

\begin{tabular}{l|c|c|c|c}
\hline Primaria & $256(87,7)$ & $19(46,3)$ & $237(94,4)$ & $<0,001$ \\
\hline Secundaria & $36(12,3)$ & $22(53,7)$ & $14(5,6)$ & \\
\hline
\end{tabular}

\section{Antecedentes familiares de $\mathrm{HH}$}

\begin{tabular}{l|l|l|l|l}
\hline Sí & $159(54,5)$ & $16(39)$ & $143(57)$ & 0,032 \\
\hline No & $133(45,5)$ & $25(61)$ & $108(43)$ & \\
\hline
\end{tabular}

Otras localizaciones de $\mathrm{HH}$

\begin{tabular}{l|c|c|c|c}
\hline Palmar & $204(69,9)$ & $3(7,3)$ & $201(80,1)$ & $<0,001$ \\
\hline Plantar & $202(69,2)$ & $3(7,3)$ & $199(79,3)$ & $<0,001$ \\
\hline Axilar & $139(47,6)$ & $7(17,1)$ & $132(52,6)$ & $<0,001$ \\
\hline Generalizada & $38(13)$ & $21(51,2)$ & $17(6,8)$ & $<0,001$ \\
\hline Tronco & $34(11,6)$ & $15(36,6)$ & $19(7,6)$ & $<0,001$ \\
\hline Cara & $93(31,8)$ & $37(90,2)$ & $56(22,3)$ & $<0,001$ \\
\hline
\end{tabular}

\section{HDSS basal}

\begin{tabular}{l|c|c|c|c}
\hline HDSS 2 & $4(1,4)$ & $1(2,4)$ & $3(1,2)$ & 0,384 \\
\hline HDSS 3 & $65(22,3)$ & $6(14,6)$ & $59(23,5)$ & \\
\hline HDSS 4 & $223(76,4)$ & $34(82,9)$ & $189(75,3)$ & \\
\hline
\end{tabular}

\section{Tratamientos previos a la $\mathrm{OxO}$}

\begin{tabular}{l|c|c|c|c}
\hline Sí & $237(81,2)$ & $19(46,3)$ & $218(86,9)$ & $<0,001$ \\
\hline No & $55(18,8)$ & $22(53,7)$ & $33(13,1)$ & \\
\hline
\end{tabular}

DE: desviación estándar; HDSS: Hyperhidrosis Disease Severity Scale; HH: hiperhidrosis; HHCC: hiperhidrosis con afectación del cuero cabelludo; HHSCC: hiperhidrosis sin afectación del cuero cabelludo; n: tamaño de la muestra; OxO: oxibutinina oral; p: nivel de significación estadística. 
Hiperhidrosis del cuero cabelludo: particularidades clínicas y de respuesta al tratamiento con oxibutinina oral

TABLA 2. Dosis media diaria, respuesta y efectos secundarios respecto al tratamiento con oxibutinina oral a los 3 y 12 meses de comenzarlo, y motivo de suspensión

\begin{tabular}{|c|c|c|}
\hline Total de pacientes & Pacientes con HHCC & Pacientes con \\
con HH tratados con & $\mathrm{n}=41$ & HHSCC \\
OxO & & $\mathrm{n}=251$ \\
$\mathrm{n}=292$ & & \\
\hline
\end{tabular}

$p$

HHSCC

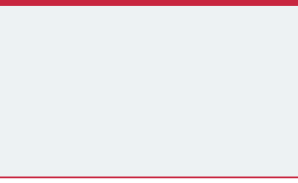

HDSS media

\begin{tabular}{l|c|c|c|c}
\hline Pretratamiento & 3,7 & 3,8 & 3,7 & 0,314 \\
\hline $\begin{array}{l}\text { Postratamiento (3 } \\
\text { meses) }\end{array}$ & 2,2 & 2,5 & 2,1 & 0,064 \\
\hline $\begin{array}{l}\text { Postratamiento (12 } \\
\text { meses) }\end{array}$ & 1,9 & 2,1 & 1,9 & 0,215 \\
\hline
\end{tabular}

Dosis media diaria de $\mathrm{OxO}$

\begin{tabular}{l|l|l|l|l}
\hline A los 3 meses & 7,6 & 7,7 & 7,6 & 0,939 \\
\hline A los 12 meses & 8,4 & 9,3 & 8,3 & 0,179 \\
\hline
\end{tabular}

Respuesta a los 3 meses

\begin{tabular}{l|c|c|c|c}
\hline Excelente & $172(60,1)$ & $21(51,2)$ & $151(61,6)$ & 0,118 \\
\hline Parcial & $63(22)$ & $8(19,5)$ & $55(22,4)$ & \\
\hline Sin cambios & $51(17,8)$ & $12(29,3)$ & $39(15,9)$ & \\
\hline
\end{tabular}

Efectos adversos a los 3 meses

\begin{tabular}{l|c|c|c|c}
\hline Sí & $200(69,2)$ & $29(70,7)$ & $171(69)$ & 0,819 \\
\hline No & $89(30,8)$ & $12(29,3)$ & $77(31)$ & \\
\hline
\end{tabular}

Xerosis oral a los 3 meses

\begin{tabular}{l|c|c|c|c}
\hline No & $122(42,2)$ & $18(43,9)$ & $104(41,9)$ & 0,868 \\
\hline Leve & $102(35,3)$ & $13(31,7)$ & $89(35,9)$ & $55(22,2)$ \\
\hline Intensa & $65(22,5)$ & $10(24,4)$ & $90(36,3)$ & 0,138 \\
\hline $\begin{array}{l}\text { Otros efectos adversos } \\
\text { a los 3 meses }\end{array}$ & $100(34,6)$ & $10(24,4)$ & & \\
\hline
\end{tabular}

Respuesta a los 12 meses

\begin{tabular}{l|c|c|c|c}
\hline Excelente & $130(71)$ & $14(66,7)$ & $116(71,6)$ & 0,232 \\
\hline Parcial & $47(25,7)$ & $5(23,8)$ & $42(25,9)$ & \\
\hline Sin cambios & $6(3,3)$ & $2(9,5)$ & $4(2,5)$ & \\
\hline
\end{tabular}

Efectos adversos a los 12 meses

\begin{tabular}{l|c|c|c|c}
\hline Sí & $132(72,1)$ & $17(81)$ & $115(71)$ & 0,337 \\
\hline No & $51(27,9)$ & $4(19)$ & $47(29)$ & \\
\hline
\end{tabular}

Continúa 
Hiperhidrosis del cuero cabelludo: particularidades clínicas y de respuesta al tratamiento con oxibutinina oral Del Boz González FJ et al.

TABLA 2. Dosis media diaria, respuesta y efectos secundarios respecto al tratamiento con oxibutinina oral a los 3 y 12 meses de comenzarlo, y motivo de suspensión (continuación)

\begin{tabular}{|c|c}
$\begin{array}{c}\text { Total de pacientes } \\
\text { con } \mathrm{HH} \text { tratados con }\end{array}$ & $\begin{array}{c}\text { Pacientes con HHCC } \\
\text { OxO } \\
\mathbf{n}=292\end{array}$ \\
\hline
\end{tabular}

Pacientes con
HHSCC
$\mathbf{n}=251$

$p$

Xerosis oral a los 12 meses

\begin{tabular}{l|c|c|c|c}
\hline No & $63(34,4)$ & $4(19)$ & $59(36,4)$ & 0,288 \\
\hline Leve & $78(42,6)$ & $11(52,4)$ & $67(41,4)$ & $36(22,2)$ \\
\hline Intensa & $42(23)$ & $6(28,6)$ & $53(32,7)$ & 0,166 \\
\hline $\begin{array}{l}\text { Otros efectos adversos } \\
\text { a los 12 meses }\end{array}$ & $57(31)$ & $4(18,2)$ & \\
\hline
\end{tabular}

Razón de abandono del tratamiento con $\mathrm{OxO}$

\begin{tabular}{l|c|c|c|c}
\hline Efectos adversos & $48(16,4)$ & $9(21,9)$ & $39(15,5)$ & 0,960 \\
\hline Ineficacia & $33(11,3)$ & $5(12,2)$ & $28(11,2)$ & \\
\hline $\begin{array}{l}\text { Efectos adversos }+ \\
\text { ineficacia }\end{array}$ & $45(15,4)$ & $7(17,1)$ & $38(15,1)$ & \\
\hline Decisión del paciente & $27(9,2)$ & $5(12,2)$ & $22(8,8)$ & \\
\hline
\end{tabular}

HDSS: Hyperhidrosis Disease Severity Scale; HH: hiperhidrosis; HHCC: hiperhidrosis con afectación del cuero cabelludo; HHSCC: hiperhidrosis sin afectación del cuero cabelludo; n: tamaño de la muestra; OxO: oxibutinina oral; $p$ : nivel de significación estadística.

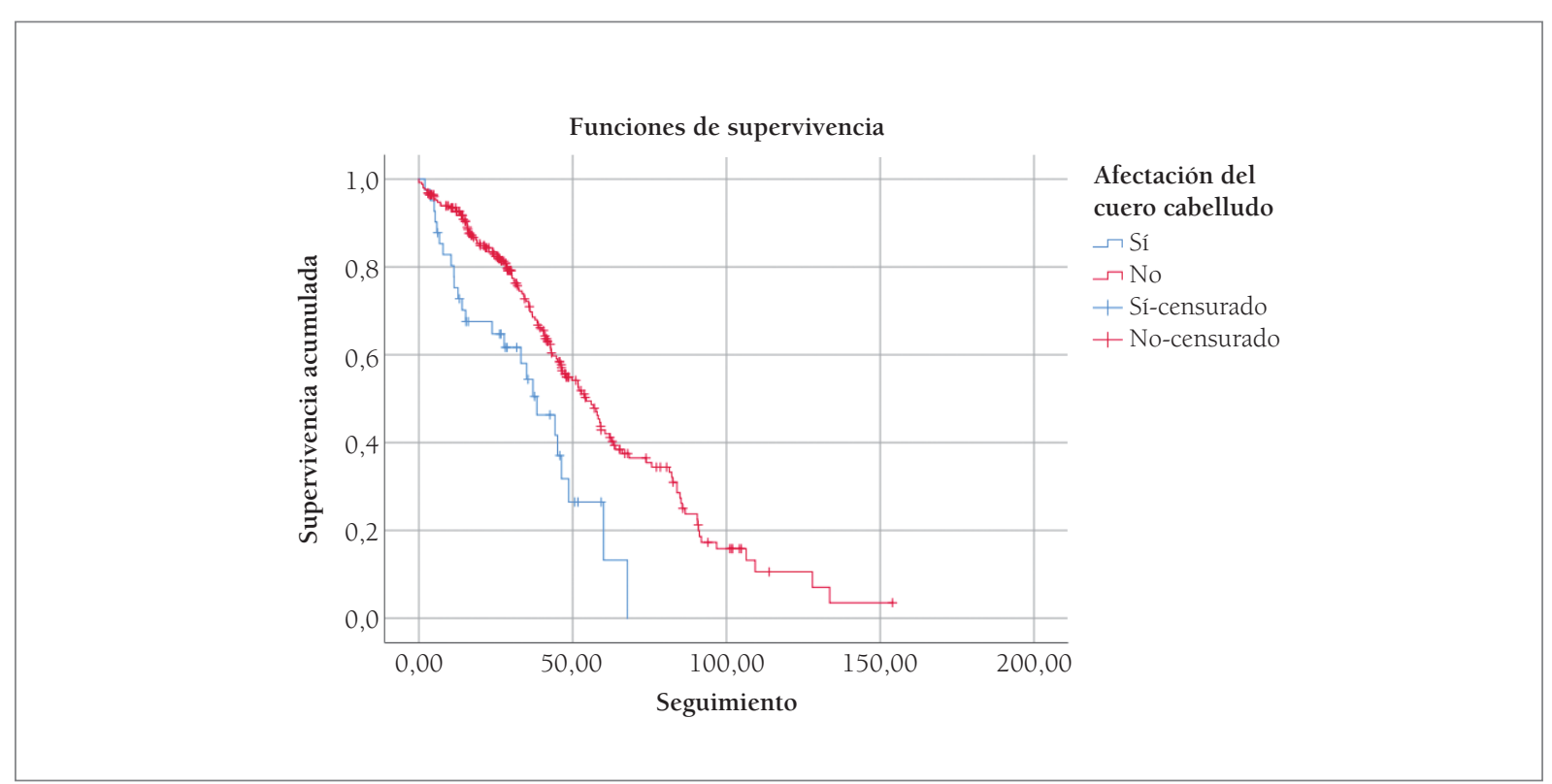

FIGURA 1. Análisis de supervivencia (curva de Kaplan-Meier) para los pacientes con hiperhidrosis con afectación del cuero cabelludo y los pacientes con hiperhidrosis sin afectación del cuero cabelludo tratados con oxibutinina oral.

La probabilidad de supervivencia se representa en el eje Y, y el tiempo de seguimiento (en meses), en el eje X. Los pacientes con hiperhidrosis del cuero cabelludo se representan en azul, y aquellos con hiperhidrosis sin afectación del cuero cabelludo, en rojo. 
edad mayor, y presentaron, sobre todo, hiperhidrosis secundaria. Además, sufrieron mayor afectación simultánea de hiperhidrosis en la cara y el tronco, y menor de hiperhidrosis asociada en las palmas, plantas y axilas; asimismo, estos pacientes cuentan con menos alternativas terapéuticas disponibles, por lo que no es de extrañar que la mayoría no hubiese realizado otros tratamientos antes de comenzar con la $\mathrm{OxO}$, aunque se observa similar respuesta y tolerancia a su uso, si bien suelen abandonar antes este tratamiento.

\section{CONCLUSIONES}

Estos resultados indican que los pacientes con HHCC, respecto a aquellos con HHSCC, son un subgrupo de pacientes con unas particularidades clínico-terapéuticas distintivas.

\section{BIBLIOGRAFÍA}

1. Nicholas R, Quddus A, Baker DM. Treatment of primary craniofacial hyperhidrosis: a systematic review. Am J Clin Dermatol. 2015;16(5):361-70

2. Eustace K, Wilson NJ. Postmenopausal craniofacial hyperhidrosis. Clin Exp Dermatol. 2018;43(2):180-2.

3. Alsharqi A, Wilson NJE. Craniofacial hyperhidrosis in postmenopausal women. Australas J Dermatol. 2012;53(2):158-9.

4. Karlqvist M, Rosell K, Rystedt A, Hymnelius K, Swartling C. Botulinum toxin B in the treatment of craniofacial hyperhidrosis. J Eur Acad Dermatol Venereol. 2014;28(10):1313-7.

5. Lehrer E, Nogues A, Jaume F, Mullol J, Alobid I. Assessment of craniofacial hyperhidrosis and flushing by sphenopalatine blockade - a randomized trial. Rhinology. 2020;58(1):51-8.

6. Anders D, Moosbauer S, Naumann MK, Hamm H. Craniofacial hyperhidrosis successfully treated with botulinum toxin type A. Eur J Dermatol. 2008;18(1):87-8.

7. Del Boz J. Systemic treatment of hyperhidrosis. Actas Dermosifiliogr. 2015;106(4):271-7.

8. Solish N, Bertucci V, Dansereau A, Hong HCH, Lynde C, Lupin $\mathrm{M}$, et al.; Canadian Hyperhidrosis Advisory Committee. A comprehensive approach to the recognition, diagnosis, and severitybased treatment of focal hyperhidrosis: recommendations of the Canadian Hyperhidrosis Advisory Committee. Dermatol Surg. 2007;33(8):908-23.

9. Walling HW. Clinical differentiation of primary from secondary hyperhidrosis. J Am Acad Dermatol. 2011;64(4):690-5. 